T. Ohsawa

Nagoya Math. J.

Vol. 129 (1993), 43-52

\title{
ON THE BERGMAN KERNEL OF \\ HYPERCONVEX DOMAINS
}

\author{
TAKEO OHSAWA
}

\section{Introduction}

Let $D$ be a bounded pseudoconvex domain in $\mathbf{C}^{n}$, and let $K_{D}(z, w)$ be the Bergman kernel function of $D$. The boundary behavior of $K_{D}(z, w)$, or that of $K_{D}(z, z)$, has attracted a lot of attention because it is closely related to the pseudoconformal geometry of $D$ and $\partial D$. For instance, the Bergman metric $\partial \bar{\partial} \log K_{D}(z, z)$ is invariant under any biholomorphic transformation of $D$, and the growth-rate of $K_{D}(z, z)$ near a given boundary point $x$ is controlled by the behavior of the Levi-form of $\partial D$ near $x$. Roughly speaking, the rank of the Levi-form at $x$ is a pseudoconformal invariant that measures the growth of $K_{D}(z, z)$ near $x$, or in other words it measures how much room is left for $L^{2}$ holomorphic functions to live near $x$ (cf. [Hö], [D], [O-1], [D-H-O]). As is well known, very deep analysis is possible for $K_{D}(z, w)$ in case $\partial D$ is $C^{\infty}$ and strongly pseudoconvex, or more generally of finite type, and as a result one extend Carathéodory's theorem to several complex variables by this approach (cf. $[\mathrm{F}],[\mathrm{C}])$. On the other hand, although there exist a lot of bounded domains with non-smooth boundaries which arise naturally in complex analysis, Teichmüller spaces for example, study of the Bergman kernel for such domains does not seem to be so advanced as in the case of smoothly bounded domains. In this respect, a recent work of S. L. Krushkal' $[\mathrm{Kr}]$ has drawn the author's attention, where it is stated that the finite dimensional Teichmüller spaces $T(g, n)$ fall into the class of hyperconvex domains in the sense of Stehlé [S], i.e. they admit bounded continuous exhaustion functions which are plurisubharmonic. Afterwards the author learned through a discussion with H. Tanigawa that, whenever the Carathéodory metric of $D$ is complete, which is the case if $D=T(g, n)$, it follows that $D$ is hyperconvex and that $K_{D}(z, z)$ is an exhaustion function of $D$ (cf. [S] and [E]). Therefore he was naturally led to ask whether these two properties, i.e. the hyperconvexity of $D$

Received June 8, 1992. 
and the exhaustiveness of $K_{D}(z, z)$, are equivalent or not. The purpose of the present paper is to settle this question completely by showing the following.

THEOREM. Let $D$ be a hyperconvex bounded domain in $\mathbf{C}^{n}$. Then $\lim _{z \rightarrow \partial D} K_{D}(z, z)=\infty$.

Proposition. There exists a domain $D \subset \mathbf{C}$ of type $(L)$ in the sense of Zalcman (cf. §3) such that $D$ is not hyperconvex but $\lim _{z \rightarrow \partial D} K_{D}(z, z)=\infty$.

The author is very grateful to his younger colleague Dr. H. Tanigawa for her helps and valuable suggestions. He owes much to her the proof of Theorem. Furthermore, from her he has learned Wiener's criterion for the Dirichlet-regularity of the boundary points of domains in $\mathbf{C}$, which was decisive for the proof of Proposition. He also thanks to Professors J. Noguchi, T. Murai, K. Diederich and Makoto Suzuki for stimulating conversations.

\section{\$1. One dimensional case}

Let $U$ be a nonempty bounded open subset of $\mathbf{C}$ that admits a continuous subharmonic function $u: U \rightarrow[-\infty, 0)$ such that the sublevel sets $\{z \in U ; u(z)$ $<c$ of $u$ are relatively compact in $U$ for all $c<0$. Any function $u$ enjoying these properties shall be called an exhaustion function of $U$ by an abuse of language. We note that if $u$ is an exhaustion function of $U$, then $\hat{u}(z):=\max \{u(z),-1\}$ is also an exhaustion function of $U$. We shall denote the set $\{z \in U ; u(z)<c\}$ by $U(u, c)$, or simply by $U_{c}$ when $u$ is understood from the context. For each $\zeta \in U$ let $S_{\zeta}$ be the set of continuous functions $v: U \rightarrow[-\infty, 0)$ such that $v(z)$ $-\log |\zeta-z|$ is subharmonic on $U$. Note that $S_{\zeta} \neq \phi$ for any $\zeta \in U$, since $\log |\zeta-z|-C \in S_{\zeta}$ whenever $C$ is a real number satisfying $\exp C>\operatorname{diam} U$. Here diam $U:=\sup \{|z-w| ; z, w \in U\}$. Then we put

$$
g_{U}(\zeta, z)=\sup \left\{v(z) ; v \in S_{\zeta}\right\}
$$

Let us recall basic facts on $g_{U}$.

Proposition 1. For each $\zeta \in U, g_{U}(\zeta, \cdot)$ is a continuous function on $U$ with values in $[-\infty, 0)$, and $g_{U}(\zeta, z)-\log |\zeta-z|$ is harmonic in $z$ on $U$.

For the proof the reader is referred to [F-K, pp. 181-183]. 
Proposition 2. $g_{U}(\zeta, z)=g_{U}(z, \zeta)$ for all $(\zeta, z) \in U \times U$.

For the proof, see [F-K, p. 186].

The following is also well known, but we shall give its proof because we need the same argument later in the case of several complex variables.

Proposition 3. Under the above situation, $g_{U}(\zeta, \cdot)$ is an exhaustion function for every $\zeta \in U$.

Proof. Let $u$ be any exhaustion function of $U$. Given any point $\zeta \in U$, choose $\varepsilon>0$ so that $U_{-3 \varepsilon} \ni \zeta$. Then we choose a $C^{\infty}$ function $\rho: U \rightarrow[0,1]$ with supp $\rho$ $\subset U_{-3 \varepsilon}$ such that $\rho(z)=1$ on a neighbourhood of $\zeta$. Let $u_{\varepsilon}$ be any $C^{\infty}$ strictly subharmonic function on $U_{-\varepsilon}$ satisfying $\sup _{z \in U_{-\varepsilon}}\left|u(z)-u_{\varepsilon}(z)\right|<\varepsilon$, which one may construct by modifying $u(z)+\delta\|z\|^{2}$ for sufficiently small $\delta>0$, and put

$$
u_{(\varepsilon)}(z)=\left\{\begin{array}{lr}
\max \left\{2 u(z), u_{\varepsilon}(z)-2 \varepsilon\right\} & \text { on } u_{-\varepsilon} \\
2 u(z) & \text { on } U \backslash U_{-\varepsilon} .
\end{array}\right.
$$

Clearly, $u_{(\varepsilon)}$ is an exhaustion function of $U$ and $u_{(\varepsilon)}$ is $C^{\infty}$ on $U_{-3 \varepsilon}$ since $u_{(\varepsilon)}=$ $u_{\varepsilon}-2 \varepsilon$ there. Hence there exists a constant $C>0$ such that

$$
C u_{(\varepsilon)}(z)+\rho(z) \log |\zeta-z| \in S_{\zeta} .
$$

Clearly this forces $g_{U}(\zeta, z)$ to be an exhaustion function of $U$.

Let $K \subset U$ be any compact subset and let $\varepsilon>0$ be chosen so that $U_{-3 \varepsilon} \supset K$, and let $u_{(\varepsilon)}$ be as in the proof of Proposition 3 . Since $u_{(\varepsilon)}$ is strictly subharmonic on $U_{-3 \varepsilon}$ there exists a constant $C=C(K, \varepsilon)>0$ such that, given any point $\zeta \in K$, one can find a neighbourhood $V \ni \zeta$ contained in $U_{-3 \varepsilon}$ and a $C^{\infty}$ function $\rho: U \rightarrow[0,1]$ satisfying supp $\rho \subset U_{-3 \varepsilon}$ and $\rho=1$ on $V$ so that

$$
C u_{(\varepsilon)}(z)+\rho(z) \log |\zeta-z| \in S_{\zeta} .
$$

Therefore, by the same reasoning as in the proof of Proposition 3 one deduces the following.

Lemma 4. For any $\varepsilon>0$ there exists a $\delta>0$ such that $g_{U}(\zeta, z)>-1$ if $\zeta \in U_{-\varepsilon}$ and $z \in U \backslash U_{-\delta}$.

Combining Lemma 4 with Proposition 2 we obtain 
PRoposition 5. For any $\varepsilon>0$ there exists a $\delta>0$ such that $g_{U}(\zeta, z)>-1$ if $\zeta \in U \backslash U_{-\delta}$ and $z \in U_{-\varepsilon}$.

Note that Proposition 5 implies in particular that the area of the sublevel set $U\left(g_{U}(\zeta, \cdot),-1\right)$ approaches zero as $\zeta \rightarrow \partial U$. We shall deduce from it by a standard $L^{2}$-method that the Bergman kernel $K_{U}(z, z)$ approaches infinity as $z \rightarrow \partial U$.

Since

$$
K_{U}(z, z)=\sup \left\{|f(z)|^{2} ; \frac{\sqrt{-1}}{2} \int_{U}|f|^{2} d z \wedge d \bar{z}=1 \text { and } \bar{\partial} f=0 \text { on } U\right\}
$$

it suffices to show that, given any sequence $\zeta_{\text {J }}$ approaching $\partial U$, one can find a family of $L^{2}$ holomorphic functions $f_{j}$ on $U$ whose $L^{2}$ norms are uniformly bounded by a constant, such that $\lim _{j \rightarrow \infty} f_{j}\left(\zeta_{j}\right)=\infty$. For that purpose we first consider a family of $C^{\infty}$ functions $g_{j}$ defined by

$$
g_{j}(z)= \begin{cases}a\left(\zeta_{j}\right)^{-1 / 2} \chi\left(g_{U}\left(\zeta_{j}, z\right)\right) & \text { for } z \neq \zeta_{j} \\ a\left(\zeta_{j}\right)^{-1 / 2} & \text { if } z=\zeta_{j}\end{cases}
$$

where $a\left(\zeta_{j}\right)$ denote the area of $U\left(g_{U}\left(\zeta_{j}, z\right),-1\right)$ and $\chi$ is a $C^{\infty}$ function on $\mathbf{R}$ such that $\chi(t)=1$ on $(-\infty,-2)$ and $\chi(t)=0$ on $[-1, \infty)$ which is fixed independent of $j$. It is clear that the $L^{2}$ norms of $g_{j}$ are bounded by 1 and that $g_{j}\left(\zeta_{j}\right) \rightarrow \infty$ as $j \rightarrow \infty$ by Proposition 5. Now we put

$$
w_{j}=\bar{\partial}_{z} g_{j} \wedge d z,
$$

where $\bar{\partial}_{z}:=d \bar{z} \frac{\partial}{\partial \bar{z}}$. Then we observe that, with respect to the pseudometric $-\partial_{z} \bar{\partial}_{z} \log \left(-g_{U}\left(\zeta_{j}, z\right)+1\right)\left(\partial_{z}:=d z \frac{\partial}{\partial z}\right)$ on $U \backslash\left\{\zeta_{j}\right\}$, the $L^{2}$ norm of $w_{j}$ on $U \backslash\left\{\zeta_{j}\right\}$ with respect to the weight $\exp \left\{-2 g_{U}\left(\zeta_{j}, z\right)+\log \left(-g_{U}\left(\zeta_{j}, z\right)+1\right)\right\}$ stays bounded as $j \rightarrow \infty$. In fact, this can be easily seen from

(1) $\partial_{z} \chi\left(g_{U}\left(\zeta_{j}, z\right)\right)=\chi^{\prime}\left(g_{U}\left(\zeta_{j}, z\right)\right) \partial_{z} g_{U}\left(\zeta_{j}, z\right)$

(2) $-\partial_{z} \bar{\partial}_{z} \log \left(-g_{U}\left(\zeta_{j}, z\right)+1\right)=\left(g_{U}\left(\zeta_{j}, z\right)-1\right)^{-2} \partial_{z} g_{U}\left(\zeta_{j}, z\right) \bar{\partial}_{z} g_{U}\left(\zeta_{j}, z\right)$

(3) $\operatorname{supp} w_{j} \subset\left\{z ;-2<g_{U}\left(\zeta_{j}, z\right)<-1\right\}$.

One may take $18 \sup \left|\chi^{\prime}\right|^{2}$ as a bound, for instance. Hence, by an $L^{2}$ cohomology vanishing theorem (cf. [O-2, Theorem 2.8]. See also [De]), there exists a $(1,0)$-form $v_{\zeta_{j}}$ on $U \backslash\left\{\zeta_{j}\right\}$ satisfying $\bar{\partial} v_{\zeta_{j}}=w_{j}$ and 
(4) $\sqrt{-1} \int_{U \backslash\left\{\zeta_{j}\right\}} \exp \left\{-2 g_{U}\left(\zeta_{j}, z\right)+\log \left(-g_{U}\left(-g_{U}\left(\zeta_{j}, z\right)+1\right)\right\} v_{\zeta_{j}} \wedge \bar{v}_{\zeta_{j}}\right.$

$$
\leq 18 \sup \left|\chi^{\prime}\right|^{2}
$$

since $U \backslash\left\{\zeta_{j}\right\}$ is a complete Kähler manifold and the relation between the fiber metric of the trivial bundle and the pseudometric on the underlying space is given by

$$
\partial_{z} \bar{\partial}_{z}\left\{-2 g_{U}\left(\zeta_{j}, z\right)+\log \left(-g_{U}\left(\zeta_{j}, z\right)\right)\right\}=\partial_{z} \bar{\partial}_{z} \log \left(-g_{U}\left(\zeta_{\jmath}, z\right)\right)
$$

Now we set

$$
f_{j}(z) d z:=g_{j}(z) d z-v_{\zeta_{j}}
$$

Then $f_{j}(z)$ is an $L^{2}$ holomorphic function on $U \backslash\left\{\zeta_{j}\right\}$ so that it extends to an $L^{2}$ holomorphic function on $U$, say $\tilde{f}_{j}(z)$. By the integrability condition (4) it is clear that $\tilde{f}_{j}\left(\zeta_{j}\right)=g_{j}\left(\zeta_{j}\right)$ and that the $L^{2}$ norms of $\tilde{f}_{j}$ are uniformly bounded. This is exactly what we wanted to show.

\section{\$2. Proof of Theorem}

Let $D$ be a bounded domain in $\mathbf{C}^{n}$ and assume that $D$ is hyperconvex, i.e. that $D$ admits a continuous plurisubharmonic function with values in $[-\infty, 0)$ say $u$, such that $D_{c}:=\{z ; u(z)<c\}$ is relatively compact in $D$ for all $c \in(-\infty, 0)$. Such $u$ shall be called an exhaustion function of $D$ for brevity. By a similar argument as in Proposition 3 we shall prove the following.

Lemma 6. Let $D$ and $u$ be as above, and let $\tilde{u}(z):=\max \{u(z),-1\}$. Then, for any $\varepsilon>0$ compact subset $K \subset D$ there exists an exhaustion function $u_{\varepsilon}$ of $D$ such that

(i ) There exists a neigbourhood of $K$ on which $u_{\varepsilon}$ is $C^{\infty}$ and strictly plurisubharmonic.

(ii) $\sup _{z \in D}\left|\tilde{u}(z)-u_{\varepsilon}(z)\right|<\varepsilon$.

Proof. Given $\varepsilon$ and $K$ as above, let us fix $c \in[-1,0)$ so that $K \subset D_{c}:=$ $\{x ; u(x)<c\}$. Then we choose a $C^{\infty}$ convex increasing function $\lambda: \mathbf{R} \rightarrow \mathbf{R}$ such that

$$
\begin{gathered}
\lambda(t)=t \text { if } t \in(c / 2, \infty) \\
\delta:=\inf \{\lambda(t)-t ; t<c\}>0
\end{gathered}
$$


and

$$
\sup \{\lambda(t)-t ; t>\inf \tilde{u}\}<\varepsilon / 2 \text {. }
$$

Then we put

$$
v(z)=\lambda(u(z))-\delta / 2 \text { for } z \in D .
$$

Since $v(z)$ is a continuous plurisubharmonic function, there exists a sequence of $C^{\infty}$ plurisubharmonic functions $\left\{v_{k}\right\}_{k=1}$ defined on a neighbourhood of $D_{c / 2}$ such that $v_{k} \rightarrow v$ uniformly on $D_{c / 2}$ as $k \rightarrow \infty$. We choose $k_{0}$ so that $v_{k_{0}}(z)>u(z)$ for all $z \in D_{c}$ and $\left|v_{k_{0}}(z)-v(z)\right|<\delta / 4$ for all $z \in D_{c / 2}$. Then we put

$$
u_{\varepsilon}(z)=\left\{\begin{array}{l}
\max \left\{v_{k_{0}}(z), u(z)\right\} \quad \text { for } \quad z \in D_{c / 2} \\
u(z) \text { for } z \in D \backslash D_{c / 2} .
\end{array}\right.
$$

It is clear that ( $\mathrm{i}$ ) and (ii) are both satisfied by $u_{\varepsilon}$.

Let $L \subset \mathbf{C}^{n}$ be any complex line intersecting with $D$. Then $L \cap D$ is an open subset of $L \cong \mathbf{C}$ so that $g_{L \cap D}(\zeta, z)$ is well defined on $L \cap D$ just as in Section one. As we have deduced Lemma 4 from the proof of Proposition 3, we obtain from Lemma 6 a similar conclusion:

PROPOSITION 7. Let $c<0$. Then there exists a $\delta>0$ such that, for any point $\zeta \in D \backslash D_{-\delta}$ and any complex line $L$ passing through $\zeta$ one has $g_{L \cap D}(\zeta, z)>-1$ for all $z \in L \cap D_{C}$.

Proof is left to the reader.

Let us denote by $\mathscr{L}\left(\mathbf{C}^{n}\right)$ the set of complex lines in $\mathbf{C}^{n}$. Given any bounded open subset $D^{\prime} \subset \mathbf{C}^{n}$ and $L \in \mathscr{L}\left(\mathbf{C}^{n}\right)$, let $A\left(L \cap D^{\prime}\right)$ denote the euclidean area of $L \cap D^{\prime}$ measured as an open subset of $L$. For any point $\zeta \in D$ and $c<0$ we put

$$
A_{c}(\zeta):=\inf \left\{A\left(L \cap D_{c}\right) ; L \in \mathscr{L}\left(\mathbf{C}^{n}\right) \text { and } \zeta \in L\right\}
$$

Clearly one has

$$
\lim _{c \nearrow 0} \sup _{\zeta \in D \backslash D_{c}} A_{c}(\zeta)=0 .
$$

Let $K_{L \cap D}(z, w)$ denote the Bergman kernel function of $L \cap D$ as a bounded domain in $L$. Then (6) together with Proposition 7 implies that

$$
\lim _{c \nearrow 0} \inf _{z \in D \backslash D_{c}} \sup \left\{K_{L \cap D}(z, z) ; L \in \mathscr{L}\left(\mathbf{C}^{n}\right) \text { and } z \in L\right\}=\infty
$$


in view of the estimate for $K_{L \cap D}(z, z)$ from below which we obtained in Section one. Hence the proof of Theorem ends if we admit the following.

Theorem 8. (cf. [O-3, Corollary 2]. See also [O-T].) Let $Y$ be a pure dimensional closed complex submanifold of $\mathbf{C}^{n}$, let $G \subset \mathbf{C}^{n}$ be a bounded domain of holomorphy, and let $u$ be any plurisubharmonic function on $G$. Then there exists a constant $A$ that depends only on $Y$ and the diameter of $G$ such that, for any holomorphic function $f$ on $G \cap Y$ satisfying

$$
\int_{Y} e^{-u}|f|^{2} d V_{Y}<\infty
$$

there exists a holomorphic extension $F$ of $f$ to $G$ such that

$$
\int_{G} e^{-u}|F|^{2} d V \leq A \int_{Y} e^{-u}|f|^{2} d V_{Y}
$$

Here $d V$ and $d V_{Y}$ denote respectively the volume elements with respect to the euclidean metric and its restriction to $Y$.

\section{§3. A counterexample}

In view of Theorem, it is natural to ask whether any bounded domain $D$ satisfying $\lim _{z \rightarrow \partial D} K_{D}(z, z)=\infty$ is hyperconvex, or not. The answer is no. We shall show it by giving a counterexample.

Definition. Let $\Delta$ be the unit disc in $\mathbf{C}$ and let $\left\{x_{k}\right\}_{k=1}^{\infty} \subset(0,1)$ be a strictly decreasing sequence of points converging to 0 . We say $D \subset \Delta$ a domain of type $(L)$ (in the sense of Zalcman [Z]) if

$$
D=\Delta \backslash \overline{\bigcup_{k=1}^{\infty} \Delta\left(x_{k}, r_{k}\right)}
$$

where $\left\{\Delta\left(x_{k}, r_{k}\right)\right\}_{k=0}^{\infty}$ is a disjoint family of discs in $\Delta$ which are centered at $x_{k}$ and with radius $r_{k}>0$.

For the sake of explicitness, we let $x_{k}=2^{-k}$ and $r_{k}=2^{-k N(k)}$, where $N(k)>2$ for all $k$, and denote the corresponding domain of type $(L)$ by $D_{N}$. Then, from Wiener's criterion for the Dirichlet-regularity of the boundary points of domains in $\mathbf{C}$, we obtain 
Proposition 9. (See [H, p. 401] and also [P] and [W].) There exists a subharmonic function $u$ on $D_{N}$ such that

( i ) $\lim _{z \rightarrow 0} u(z)=0$

(ii) $\sup \left\{u(z) ; z \in D_{N}\right.$ and $\left.|z|>\varepsilon\right\}<0$ for all $\varepsilon>0$

if and only if $\sum_{k=1}^{\infty} N(k)^{-1}=\infty$.

It is easy to see from Proposition 9 that $D_{N}$ is hyperconvex if and only if $\sum_{k=1}^{\infty} N(k)^{-1}=\infty$. We are now interested in the situation where the sum of $N(k)^{-1}$ is finite, and going to prove the following.

Lemma 10. Let $D_{N}$ be a domain of type $(L)$ defined as above. Suppose $N$ satisfies

$$
\sum_{k=1}^{\infty}(k N(k))^{-1 / 2}<\infty
$$

and

$$
\lim _{r \searrow 0} \sum_{k=1}^{\infty}\left\{k N(k)\left(r+2^{-k}\right)\right\}^{-1}=\infty .
$$

Then $\lim _{z \rightarrow \partial D_{N}} K_{D_{N}}(z, z)=\infty$.

Proof. Let $z_{0} \in \partial D_{N}$ be any point and express it as

$$
z_{0}=2^{-k}+\left(\rho_{k}+2^{-k N(k)}\right) \exp \left(\sqrt{-1} \theta_{k}\right)\left(\rho_{k} \geq 0 \text { and } \theta_{k} \in[0,2 \pi)\right),
$$

for $k=1,2, \cdots$.

Let us define a holomorphic function $f_{k}$ on $D_{N}$ by

$$
f_{k}(z)=\exp \left(\sqrt{-1} \theta_{k}\right)\left\{\sqrt{k N(k)}\left(z-2^{-k}\right)\right\}^{-1} .
$$

Then

$$
f_{k}\left(z_{0}\right)=\left(\sqrt{k N(k)}\left(\rho_{k}+2^{-k N(k)}\right)\right\}^{-1}
$$

and, for the $L^{2}$ norms $\left\|f_{k}\right\|$ of $f_{k}$ we have

$$
\sup _{k}\left\|f_{k}\right\|^{2} \leq 4 \pi \text {. }
$$

Let us put 


$$
g_{z_{0}}(z)=\sum_{k=1}^{\infty}(k N(k))^{-1 / 2} f_{k}(z) .
$$

Then $\left\|g_{z_{0}}(z)\right\| \leq 4 \pi \sum_{k=1}^{\infty}(k N(k))^{-1 / 2}=$ const $<\infty$ by (8) and

$$
\begin{aligned}
g_{z_{0}}\left(z_{0}\right) & =\sum_{k=1}^{\infty}\left(k N(k)\left(\rho_{k}+2^{-k N(k)}\right)\right)^{-1} \\
& >\sum_{k=1}^{\infty}\left(k N(k)\left(\left|z_{0}\right|+2^{-k}\right)\right)^{-1} .
\end{aligned}
$$

Thus, for any sequence $\left\{z_{j}\right\}_{j=0}^{\infty} \subset D_{N}$ with $\lim _{j \rightarrow \infty} z_{j}=0$ we obtain

$$
\lim _{j \rightarrow \infty} g_{z_{j}}\left(z_{j}\right)=\infty
$$

by (9). This shows in particular that $\lim _{z \rightarrow 0} K_{D_{N}}(z, z)=\infty$. Since $\partial D_{N} \backslash\{0\}$ is a disjoint union of circles it is clear that $\lim _{z \rightarrow y} K_{D_{N}}(z, z)=\infty$ for all $y \in \partial D_{N} \backslash\{0\}$. Therefore we can conclude that $\lim _{z \rightarrow \partial D_{N}} K_{D_{N}}(z, z)=\infty$.

To see the validity of Proposition stated in the introduction, one may take $k^{2}$ as $N(k)$. Then $D_{N}$ is not hyperconvex from Proposition 9 and $\lim _{z \rightarrow \partial D_{N}} K_{D_{N}}(z, z)=\infty$ by Lemma 10 .

\section{REFERENCES}

[C] Catlin, D., Subelliptic estimates for the $\bar{\partial}$-Neumann problem on pseudoconvex domains, Ann. of Math., 126 (1986), 131-191.

[De] Demailly, J. P., Estimation $L^{2}$ pour l'operateur $\bar{\partial}$ d'un fibrè vectoriel holomorphe semi-positif au-dessus d'une variété kählérienne complète, Ann. sc. Ec. Norm. Sup., 15 (1982), 457-511.

[D] Diederich, K., Das Randverhalten der Bergmanschen Kernfunktion und Metrik in streng pseudokonvexen Gebieten, Math. Ann., 189 (1970), 9-36.

[D-H-O] Diedreich, K., Herbort, G. and Ohsawa, T., The Bergman kernel on uniformly extendable pseudoconvex domains, Math. Ann., 273 (1986), 471-478.

[E] Earle, C. J., On the Carathéodory metric in Teichmüller spaces, in Discontinuous groups and Riemann surfaces, Ann. Math. Stud., 79, Princeton University Press, 1974.

[F-K] Farkas, H. M. and Kra, I., Riemann surfaces, Springer-Verlag, 1991.

[F] Fefferman, C., The Bergman kernel and biholomorphic mappings of pseudoconvex domains, Invent. Math., 26 (1974), 1-65.

[H] Hayman, W. K., Subharmonic functions Vol. 2, London Math. Soc. Monograph No. 20, Academic Press 1989.

[Hö] Hörmander, L., $L^{2}$-estimates and existence theorems for the $\bar{\partial}$-operator, Acta Math., 113 (1965), 89-152.

[Kr] Krushkal', S. L., Strengthening pseudoconvexity of finite-dimensional Teichmüller spaces, Math. Ann., 290 (1991), 681-687. 
[O-1] Ohsawa, T., Boundary behavior of the Bergman kernel function on pseudoconvex domains, Publ. RIMS, Kyoto Univ., 20 (1984), 897-902.

[O-2] - Vanishing theorems on complete Kähler manifolds, Publ. RIMS, Kyoto Univ., 20 (1984), 21-38.

[O-3] - On the extension of $L^{2}$ holomorphic functions II, Publ. RIMS, Kyoto Univ., 24 (1988), 265-274.

[O-T] Ohsawa, T. and Takegoshi, K., On the extension of $L^{2}$ holomorphic functions, Math. Z., 197 (1988), 1-12.

[P] Poincaré, H., Théorie du potentiel Newtonien, Leçons professées à la Sorbonne, Paris, 1899.

[S] Stehlé, J. L., Fonctions plurisubharmoniques et convexite holomorphe de certaines fibrès analytiques, C. R. Hebd. Acad. Sci. Ser. A-B 279 (1964), $235-238$.

[W] Wiener, N., Certain notions in potential theory, J. Math. Mass. Inst. Tech., 3 (1924), 24-51.

[Z] Zalcman, L., Bounded analytic functions on domains of infinite connectivity, Trans. Amer. Math. Soc., 144 (1969), 241-269.

Department of Mathematics

School of Science

Nagoya University

Chikusa-ku, Nagoya 464-01

Japan 